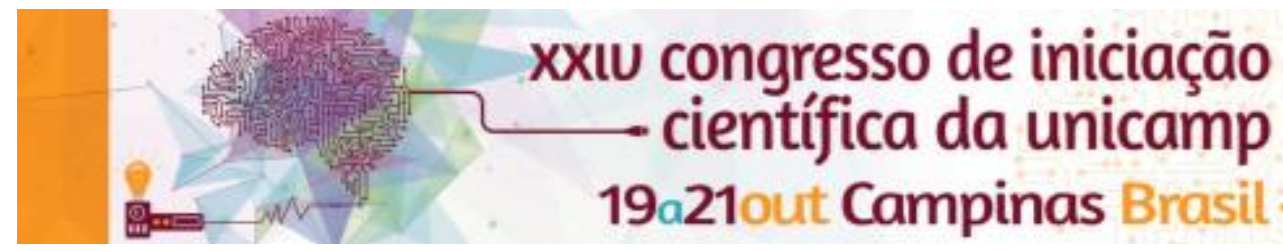

\title{
Characterization of methylation profile during progression of Wilms tumors
}

\section{João V. S. Guerra*, Rafaela M. de Azevedo, Paulo A. Faria, Beatriz de Camargo, Mariana Maschietto.}

\begin{abstract}
Wilms tumors (WTs) are the most common renal tumors with a relapse rate of $\sim 25 \%$, from which long-term survival remains approximately $50 \%$. The ability of relapsing is associated with resistance to chemotherapy and capacity of forming distant metastasis. Because in DNA methylation has being correlated with tumor cells plasticity, we purposed to characterize DNA methylation changes in normal kidney (NK), WT and distant metastasis to identify the mechanisms involved with tumor progression and resistance.
\end{abstract}

\section{Key words: \\ Wilms Tumor, DNA methylation, metastasis.}

\section{Introduction}

Wilms tumors (WTs) are embryonic tumors of the kidney composed by blastemal, epithelial and stromal components. In pre-treated tumors, blastemal predominance is associated with high risk of relapsing. Heterogeneity is also observed at molecular level, although its impact remains to be addressed. DNA methylation dictates cell plasticity including the epithelialmesenchymal transition (EMT), which is the first step for the formation of metastasis. Mutations in known genes are found in overlap in up to $30 \%$ of the cases, and loss of imprinting (LOI) of 11p15, resulting in IGF2 overexpression, is present in $70 \%$ of cases. In addition, WTs present changes in DNA methylation with tumorspecific methylated DNA being released into the blood circulation, which could be used to improve the accuracy of determining tumor response to treatment and relapse.

To identify the mechanisms involved with metastasis formation in WT, we performed comprehensive DNA methylation analyzes in matched WT, NK and metastasis.

\section{Results and Discussion}

From 110 sporadic WT cases treated in the National Institute of Cancer (INCA) between 2003 and 2014, we selected 10 matched trios (NK, WT, metastasis) with confirmed histology in formalin fixed paraffin embedded samples. Bisulfite-converted DNA was hybridized to the Infinium HumanMethylation450 BeadChip (Illumina). Seven matched trios passed all quality control steps and IDAT files were processed using Minfi package and normalized with SWAN method. Data were corrected for cell heterogeneity using reFACTor package. Considering the global methylation pattern, we observed that WT is hypomethylated compared to NK, and both are hypomethylated in relation to metastasis.

Limma was used to generate an empirical Bayesian framework linear model considering intrapatients and inter-groups comparisons. There were common 146 differentially methylated positions (DMPs, adj $\mathrm{P}<0.05$ ) between Tumor-Kidney (total of 296 DMPs) and Metastasis-Kidney (total of 2,620 DMPs) comparisons, with 37 hypomethylated and 109 hypermethylated $\mathrm{CpG}$ sites.
Metastasis presented more subtle changes in relation to the tumors. Therefore, we applied a direct comparison between metastasis and WT, using $p<0.05$ and methylation changes $>10 \%$ as parameters. There were 2,448 DMPs, with most of the 1,126 hypomethylated $\mathrm{CpGs}$ located in open sea and most of the 1,322 hypermethylated CpGs located in CpG islands and $\mathrm{N}$-Shelves, which are related to repression of transcription. The 2,448 DMPs were related to 1,304 genes, enriched within signaling pathways related to EMT, metabolism and immune system as well as MAPK and Wnt signaling pathways $(\operatorname{adj} P<0.05$ and 10 genes minimum). These genes are also enriched in several types of cancers, including renal tumors (adjP<0.05). Among others, alterations in PAX2, WT1 and IGF2 were previously described in WT as well as their involvement in early nephrogenesis.

\section{Conclusions}

Methylation profiles vary significantly in NK, WT and metastasis revealing an hypermethylated state for metastatic samples. CpG sites more methylated in metastatic samples are related to repression of transcription, in agreement with other studies that described an hypermethylated state in advanced cancers. We found that genes associated to the DMPs are involved with cellular pathways associated with EMT, and consequently, metastasis. As a proof of concept, genes with a role in nephrogenesis and disrupted in WT appeared as differentially methylated in our analysis.

\section{Acknowledgement}

This work is supported by FAPESP 2014/10250-7, 2015/22758-8 and 2015/06281-7.

Scott, R.H. et al. Stratification of Wilms tumor by genetic and epigenetic analy sis. Oncotarget. 2012, 3(3), 327-335.

Dome J. S. et al. Advances in Wilms tumor treatmente and biology: progress through international collaboration. Journal of Clinical Oncology. 2015, 33 (27), 2999-3007.

http://genomebiology.biomedcentral.com/articles/10.1186/s13059-014-0434-y]

Kiesslich, T. et al. Epigenetic control of epithelial-mesenchymal-transition in human cancer. Molecular and clinical oncology. 2013, 1(1), 3-11. 